Article

\title{
Sudden Event Monitoring of Civil Infrastructure Using Demand-Based Wireless Smart Sensors
}

\author{
Yuguang Fu ${ }^{1, *}$, Tu Hoang ${ }^{1}$, Kirill Mechitov ${ }^{2}$, Jong R. Kim ${ }^{3}$, Dichuan Zhang ${ }^{3}$ \\ and Billie F. Spencer, Jr. ${ }^{1}$ \\ 1 Department of Civil and Environmental Engineering, University of Illinois at Urbana-Champaign, Urbana, \\ IL 61801, USA; tuhoang2@illinois.edu (T.H.); bfs@illinois.edu (B.F.S.J.) \\ 2 Department of Computer Science, University of Illinois at Urbana-Champaign, Urbana, IL 61801, USA; \\ mechitov@illinois.edu \\ 3 Department of Civil and Environmental Engineering, Nazarbayev University, Astana 010000, Kazakhstan; \\ jong.kim@nu.edu.kz (J.R.K.); dichuan.zhang@nu.edu.kz (D.Z.) \\ * Correspondence: yfu15@illinois.edu
}

Received: 18 November 2018; Accepted: 14 December 2018; Published: 18 December 2018

check for updates

\begin{abstract}
Wireless smart sensors (WSS) have been proposed as an effective means to reduce the high cost of wired structural health monitoring systems. However, many damage scenarios for civil infrastructure involve sudden events, such as strong earthquakes, which can result in damage or even failure in a matter of seconds. Wireless monitoring systems typically employ duty cycling to reduce power consumption; hence, they will miss such events if they are in power-saving sleep mode when the events occur. This paper develops a demand-based WSS to meet the requirements of sudden event monitoring with minimal power budget and low response latency, without sacrificing high-fidelity measurements or risking a loss of critical information. In the proposed WSS, a programmable event-based switch is implemented utilizing a low-power trigger accelerometer; the switch is integrated in a high-fidelity sensor platform. Particularly, the approach can rapidly turn on the WSS upon the occurrence of a sudden event and seamlessly transition from low-power acceleration measurement to high-fidelity data acquisition. The capabilities of the proposed WSS are validated through laboratory and field experiments. The results show that the proposed approach is able to capture the occurrence of sudden events and provide high-fidelity data for structural condition assessment in an efficient manner.
\end{abstract}

Keywords: sudden event monitoring; wireless smart sensors; demand-based nodes; event-triggered sensing; data fusion

\section{Introduction}

Many civil infrastructure damage scenarios involve sudden events, such as natural disasters (e.g., earthquakes) and human-induced hazards (e.g., collisions, explosions, acts of terrorism). The occurrence of these events is generally unpredictable, and the consequences can be catastrophic. A typical example of catastrophic sudden event is found in the accidental collision between barges and a piling of railroad bridge in Mobile, Alabama, in 1993 [1]. As a result, the railroad bridge was damaged and gave way 20 min later when an Amtrak train crossed, killing 47 people. If this collision had been detected immediately and timely structural assessment of the bridge made, then the deaths of these individuals may have been prevented.

To mitigate the consequences of sudden events, the development of monitoring systems is of great importance. Traditional monitoring systems use wired sensors [2-5]. These monitoring systems not only enable sudden event detection but can also facilitate rapid condition assessment of civil 
infrastructure. Such wired monitoring systems require line power to operate and can be expensive for many large-scale structures, due primarily to high installation costs [6], often ranging from $\$ 5 \mathrm{~K}$ to $\$ 20 \mathrm{~K}$ per channel (e.g., the Bill Emerson Memorial Bridge monitoring system cost a total of $\$ 1.3 \mathrm{M}$ for 86 sensors [4,7]).

High-fidelity wireless sensors offer tremendous opportunities to reduce costs and realize the promise of pervasive sensing for structural condition assessment. However, sudden event detection using wireless sensors remains elusive. For example, the monitoring system installed on the Golden Gate Bridge was unable to detect the three earthquakes that occurred during the three-month monitoring deployment [8]. Two main challenges to detect sudden events are apparent:

(i) Limited power. Most wireless sensors are duty-cycled to preserve limited battery power; as a result, wireless sensors will miss the occurrence of sudden events when they are in power-saving sleep mode. Because the duty cycle is typically below 5\% [9], this scenario is quite likely to occur.

(ii) Response latency. Response of wireless smart sensors (WSS) from sleep mode to data acquisition may take over a second, resulting in the loss of critical information in short-duration events (e.g., earthquakes and collisions). Moreover, even if awake, sensors may be busy with other tasks (e.g., data transmission); therefore, they will be unable to respond immediately to the occurrence of sudden events, and hence miss the short-duration events.

Addressing these challenges is critical to realizing a WSS for sudden event detection.

One intuitive strategy is to provide sustainable power for WSS to enable continuous monitoring of structures subjected to sudden events, emulating traditional wired monitoring systems. For example, in 2011, Potenza et al. [10] installed a wireless structural health monitoring (SHM) system consisting of 17 WSS on a historical church, which was damaged during the 2009 L'Aquila earthquake. The nodes were powered by the existing electrical lines, which guaranteed the continuity of operation and successfully detected several earthquakes over a 3-year monitoring period. Their strategy of using electrical lines to power WSS does not retain the inherent advantages of cable removal, and thus is not practical for other sudden event monitoring applications using WSS. Energy harvesting and wireless power transfer technologies also do not provide an efficient solution. Although technologies such as solar and wind energy harvesting have been developed and validated to power WSS for periodic monitoring [11-13], the challenge is that energy harvesting from the ambient environment is intermittent and time-varying, which is not reliable to support continuous monitoring of structures. Radio frequency (RF) energy transfer and harvesting is another wireless power technique in which WSS convert the received RF signals into electricity. The energy can be transferred reliably over a distance from a dedicated energy source to each node, or dynamically exchanged between different nodes [14]. However, the energy harvesting rate is on the order of micro-watts with low efficiency [15] and is insufficient for high-power high-fidelity monitoring of sudden events.

On the other hand, power consumption can be reduced by employing various energy-saving mechanisms, which help to mitigate, but do not fully address the challenge of limited power for WSS. For example, Jalsan et al. [16] proposed layout optimization strategies for wireless sensor networks to prolong the network lifetime by optimizing communication schemes without compromising information quality. Other examples of energy-saving mechanisms include data reduction, radio optimization, and energy-efficient routing. More detailed discussion can be found in Reference [17]. Most of these strategies are designed to reduce power consumption for wireless transmission, which does not help energy conservation for continuous sensing, because most of the power draw comes from the always-on sensor.

Recent developments in event-triggered sensing present both opportunities and challenges to realize sudden event monitoring using WSS. In event-triggered sensing, WSS only initiates measurement in response to signaling of events, which helps to save both energy and memory resources, and thus prolong the lifetime of WSS. Research has been conducted to implement low-power 
components (sensors and radios) that enable continuous operation and triggering mechanisms inside each sensor node. Lu et al. [18] designed the TelosW platform, which is an upgrade of the TelosB platform [19], by adding ultra-low power wake-on sensors and wake-on radios. The wake-on sensor is able to wake up the microcontroller (MCU) on occurrence of events with a predetermined threshold. Additionally, the wake-on radio can wake up the MCU when a triggering radio message is received. Similarly, Sutton et al. [20] presented a heterogeneous system architecture which included a low-power event detector circuit and low-power wake-up receivers. Although these two technologies achieve low power consumption, they do not satisfy the high-fidelity requirement of sudden event monitoring for civil infrastructure. For example, the TelosW's analog to digital converter has only 12-bit resolution. Event-triggered sensing is also developed and implemented to facilitate railway bridge monitoring, because strain cycles and vibrations induced by trains are the most important data for bridge condition assessment (e.g., fatigue), but the arrival time of trains is generally unpredictable. Bischoff et al. [21] deployed a wireless monitoring system which provided strain measurement and fatigue assessment of the Keraesjokk Railway Bridge. Each node was triggered independently by a low-power microelectromechanical systems (MEMS) accelerometer which operated continuously and detected an approaching train. Bias due to the transient start-up nature of the strain gage was removed by a post-processing technique. Liu et al. [22] developed an on-demand sensing system, named ECOVIBE, to monitor train-induced bridge vibrations. In each wireless node, a passive event detection circuit was designed to monitor bridge vibration with no power consumption, and another adaptive logical control circuit powered off the node once the designated tasks were finished. While effective for some applications, all the aforementioned approaches will lose critical data between the occurrence of the event and the time that data begins to be collected. Conversely, response times of wireless sensors from a cold boot to data acquisition are typically well over a second, making this problem particularly acute for short-duration sudden events (e.g., impacts can last only fractions of a second).

Moving the triggering mechanism to outside the sensor nodes provides a solution to address the challenge of data loss. In general, a separate trigger node or system is used to monitor the events continuously and notify of events to sensor nodes which are in power-saving mode most of the time. The trigger node/system is required to send notifications with a certain amount of time before the arrival of events at the structure, compensating the response latency of other sensor nodes. For example, an event-driven wireless strain monitoring system was implemented on a riveted steel railway bridge near Wila, Switzerland [23]. Two trigger nodes, referred to as sentinel nodes, were placed at 50 and $85 \mathrm{~m}$ away from the bridge, detecting approaching trains and sending alarm messages using a reliable flooding protocol to wake up sensor nodes on the bridge, before the train arrived. In a 47-day deployment, the system successfully detected $99.7 \%$ of train-crossing events. Likewise, in order to detect earthquakes and initiate seismic structural monitoring, Hung et al. [24] developed an intelligent wireless sensor network embedded with an earthquake early warning (EEW) system which was able to detect $\mathrm{P}$-waves before earthquakes arrived. In addition, each sensor node was implemented with a wake-on radio which supported ultralow-power periodic listening of wake-up commands, while the main sensor node was in deep sleep mode. Once the P-wave was detected, the gateway node, integrated with the EEW system, sent wake-up commands to sensor nodes approximately $2 \mathrm{~s}$ ahead of earthquakes. Subsequently, sensor nodes started measurement with a latency time of only $229 \mathrm{~ms}$. Despite successful detection of train-crossing and seismic events, the aforementioned methods do not provide a universal solution to address the challenge of data loss for many other sudden events, e.g., bridge impact by over-height trucks and ships which can hardly be detected ahead of impacts.

In addition, some progress has been made in addressing the challenge of response latency to sudden events, when WSS are awake but not in sensing mode. Cheng \& Pakzad [8] proposed a pulse-based media access control protocol. When an earthquake occurs, a trigger message with high priority is propagated from an observation site across the WSS network to preempt current tasks; sensors will be forced to conduct measurement to capture the structural response under the earthquake. Dorvash et al. [25] developed the Sandwich node to reduce the response latency for unexpected events. 
A smart trigger node continuously measures the structural response; it will broadcast a proper message across a network of Sandwich nodes in the case of occurrence of events. Sandwich nodes keep listening to the trigger message; they will preempt current tasks once the trigger message is received. Response delay of Sandwich nodes is around $8 \mathrm{~ms}$ to the occurrence of events. Although response delay is reduced in these two strategies, the wireless sensor's radio must always be on to listen for messages from a trigger node. Unless employing an ultralow-power wake-up radio, these strategies will result in a significant power draw.

This paper proposes a new approach for monitoring civil infrastructure subjected to sudden events, aimed at detecting sudden events of any duration and capturing complete transient response of any length. A demand-based wireless smart sensor (WSS) is developed that can capture data during the sudden event that is suitable for rapid condition assessment of civil infrastructure. As opposed to periodic monitoring, the demand-based WSS only wakes up and initiates sensing in response to specific conditions, such as sudden events. The results of laboratory experiments and a field experiment show that our proposed approach can capture the occurrence of sudden events and provide high-fidelity data for structural condition assessment in a timely and power-efficient manner.

\section{Demand-Based WSS}

As discussed in the previous section, the primary issues that must be overcome to use wireless sensors to monitor civil infrastructure subjected to sudden events are: (i) the sensor must operate on battery power, (ii) high-fidelity data appropriate for SHM application must be obtained, (iii) data surrounding the occurrence of sudden events must not be lost, and (iv) the WSS node must have sufficient computational power to translate the data collected into actionable information. This section describes a demand-based WSS system that can address these issues.

\subsection{Ultralow-Power Trigger Accelerometer for Continuous Monitoring}

To ensure that the occurrence of sudden events is not missed, the monitoring system must be continuously in an on state. A wireless node that is always on would quickly deplete its battery. Therefore, the solution proposed herein is to use an ultralow-power trigger accelerometer that can continuously monitor the vibration of structures; the data from the accelerometer is stored in a First-In-First-Out (FIFO) buffer. When an event occurs, the data in the FIFO buffer will be frozen, and the sensor triggers an interrupt signal to wake up the main sensor platform and start sensing. Such a trigger accelerometer should have low power consumption to enable continuous monitoring for several years, good sensing characteristics, including a high sampling rate and adequate resolution, and a large FIFO buffer to ensure data is not lost after the triggering event.

Trigger accelerometers in the market today were compared and the candidates that satisfied the basic needs of sudden event monitoring are listed in Table 1. The power consumption reported in the table correspond to the ultralow-noise mode of each sensor. More specifically, the ADXL362, developed by Analog Devices, consumes much less power than the other trigger accelerometers. The ADXL372, an updated high-g version of ADXL362, has a larger sampling rate and measurement range, but with a sensing resolution of only $100 \mathrm{mg}$. The LIS3DSH from STMicromechanics has high resolution of $0.06 \mathrm{mg}$, but it has a high-power draw and an inadequate FIFO buffer. Finally, the MPU6050 developed by InvenSense features a large FIFO buffer and high resolution, but it consumes substantial power.

In sum, based on application needs, the ADXL362 has been selected for this study (Figure 1); it integrates a three-axis microelectromechanical systems (MEMS) accelerometer with a temperature sensor, an analog-to-digital converter, and a Serial Peripheral Interface (SPI) digital interface. The ADXL362 consumes only $13 \mathrm{uA}$ in ultralow-noise mode at $3.3 \mathrm{~V}$, which theoretically could work continuously for over two years on a single coin-cell battery. A sampling rate up to $400 \mathrm{~Hz}$ and a resolution of $1 \mathrm{mg}$ is supported, satisfying many SHM applications. The large FIFO buffer allows the sensor to save up to 512 samples, which corresponds to $1.7 \mathrm{~s}$ for all three axes sampled at $100 \mathrm{~Hz}$. 
Moreover, it has built-in logic for acceleration threshold detection; a detected event can be used as a trigger to wake up the primary sensor node.

Table 1. Comparison of trigger accelerometers in the market [26-29].

\begin{tabular}{ccccc}
\hline & ADXL362 & ADXL372 & LIS3DSH & MPU6050 \\
\hline Manufactures & Analog devices & Analog devices & STMicroelectronics & InvenSense \\
Supply voltage $(\mathrm{V})$ & $1.6-3.5$ & $1.6-3.5$ & $1.7-3.6$ & $2.4-3.5$ \\
Power consumption $(\mathrm{uA})$ & 13 & 33 & 225 & 500 \\
Sampling rate $(\mathrm{Hz})$ & $12.5 \sim 400$ & $400 \sim 6400$ & $3.125 \sim 1600$ & $4 \sim 1000$ \\
Measurement range $(\mathrm{g})$ & $\pm 2, \pm 4, \pm 8$ & \pm 200 & $\pm 2, \pm 4, \pm 8, \pm 16$ & $\pm 2, \pm 4, \pm 8, \pm 16$ \\
Resolution $(\mathrm{mg})$ & $1 \mathrm{mg}$ & $100 \mathrm{mg}$ & $0.06 \mathrm{mg}$ & $0.06 \mathrm{mg}$ \\
Spectral noise $(\mu \mathrm{g} / \sqrt{ } \mathrm{Hz})$ & $175-350$ & 5300 & 150 & 400 \\
Buffer size $(\mathrm{samples})$ & 512 & 512 & 32 & 512 \\
\hline
\end{tabular}

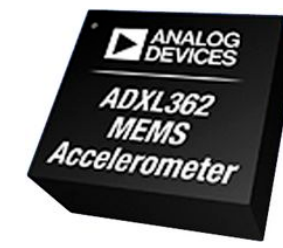

(a)

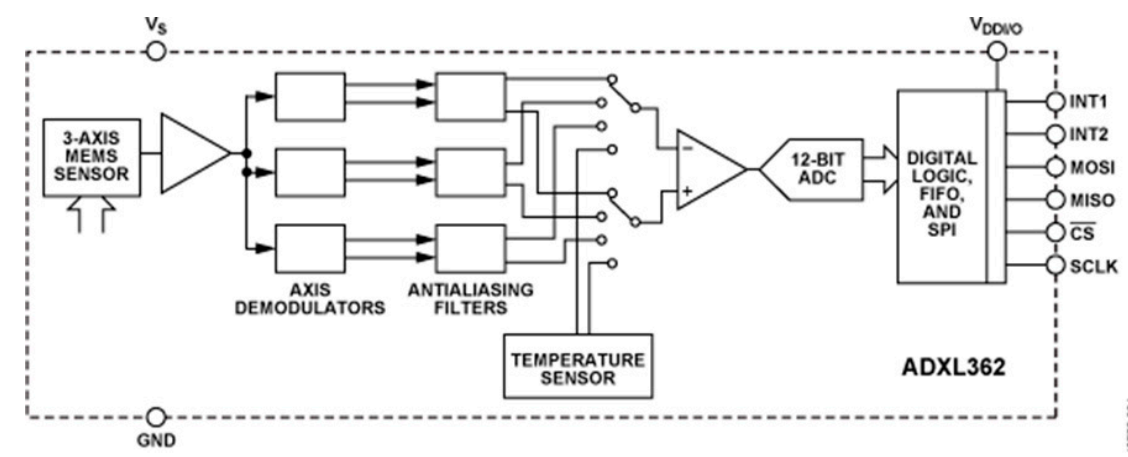

(b)

Figure 1. ADXL362: (a) sensor chip; (b) Functional block diagram [26].

\subsection{High-Fidelity Sensor Platform for Sudden Event Monitoring}

To provide high-quality measurement data and enable rapid condition assessment of structures subjected to sudden events, the sensor platform should have following features: (i) sensors and a data acquisition system that can obtain high-quality data at a high sampling rate for the event; (ii) powerful microcontroller to acquire and analyze sensor data in near real time. Other important features include: reliable communication, open-source software, and efficient data and power management.

A summary of the most advanced wireless sensor platforms available in the market currently is given in Table 2. The Xnode, developed by Embedor Technology, has a 24-bit Analog-to-Digital Converter (ADC), which is the best in its class. The microprocessor unit (MCU) of Waspmote (Libelium, Zaragoza, Spain) is not able to support rapid processing of large amount of data. The MCU information for the AX-3D (BeanAir, Berlin, Germany) and the G-Link-200 (LORD Sensing, Williston, VT, USA) is unavailable, and the operating systems of these platforms are proprietary. Note that several high-performance wireless sensor platforms (e.g., Imote2) are no longer commercially available, and hence not compared herein.

Table 2. Comparison of most advanced wireless sensor platforms in the market [30-34].

\begin{tabular}{|c|c|c|c|c|c|}
\hline & Martlet & $A X-3 D$ & Waspmote & G-Link-200 & Xnode \\
\hline ADC resolution (bits) & 12 & 16 & 16 & 20 & 24 \\
\hline Sampling rate $(\mathrm{Hz})$ & up to $3 \mathrm{M}$ & $3.5 \mathrm{k}$ & $0.5-1 \mathrm{k}$ & $0-4 \mathrm{k}$ & $0-1.6 \mathrm{k}$ \\
\hline LOS range $(\mathrm{m})$ & 500 & 650 & $1.6 \mathrm{k}$ & $2 \mathrm{k}$ & $1 \mathrm{k}$ \\
\hline Internal power source & dry cell battery & lithium-ion battery & lithium-ion battery & dry cell battery & 1thium-ion battery \\
\hline External power source & NA & primary cell/8-28 V DC & $7 \mathrm{~V} \mathrm{DC}$ & NA & $5 \mathrm{VDC}$ \\
\hline
\end{tabular}


Because of its high sensing resolution, high sampling rate, powerful microprocessor, and open-source software, the Xnode Smart Sensor [34] has been selected as the host wireless sensor platform in this study. The standard Xnode consists of three modular printed circuit boards (PCB): (i) the processor board, (ii) the radio/power board, and (iii) the sensor board (Figure 2). In particular, it employs an 8-channel, 24-bit ADC (Texas Instruments ADS131E8), allowing a maximum sampling rate up to $16 \mathrm{kHz}$ and an NXP LPC4357 microcontroller operating at frequencies up to $204 \mathrm{MHz}$, which can be used to execute data-intensive on-board computation. Moreover, it implements open-source middleware services [35], which facilitates custom application development. In addition, it possesses two SPI controllers, making it possible to communicate with the selected trigger accelerometer, the ADXL362.

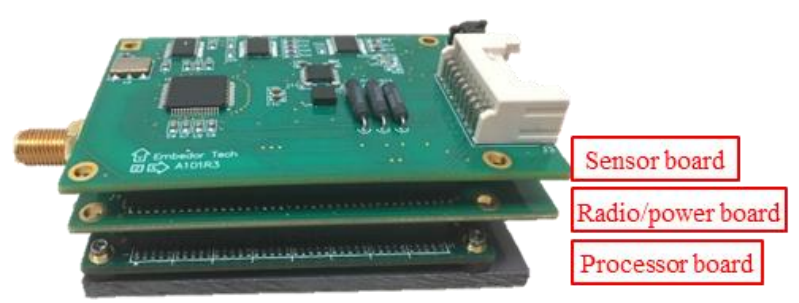

(a)

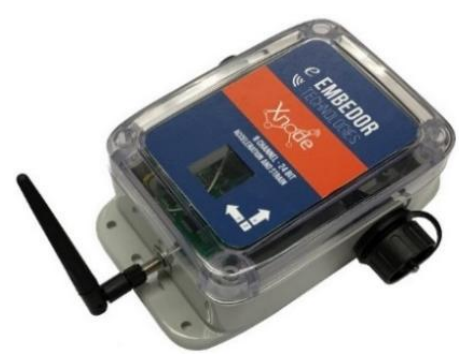

(b)

Figure 2. Xnode: (a) stacked modular boards; (b) weather-proof enclosure [34].

\subsection{Integration of Wake-up Sensor and High-Fidelity Sensor Platform}

To capture the entire event without loss of critical information, the ADXL362 accelerometer and the Xnode must be carefully integrated to build a demand-based WSS. This integration is discussed in the remainder of this section, in terms of hardware, software, and digital signal processing.

\subsubsection{Hardware Consideration}

To address the challenge of physical integration of the ADXL362 accelerometer into the Xnode, a programmable event-based switch was designed and implemented on the radio/power board of the Xnode in the demand-based WSS.

When a sudden event occurs, and the vibration exceeds a user-defined threshold, an interrupt pin in the ADXL362 generates a triggering signal. This signal is connected to a MOSFET to flip its state, turning on the Xnode and initiating high-fidelity sensing. When the event ends (lack of acceleration above a threshold), the other interrupt pin in the ADXL362 generates a signal to notify the Xnode to stop high-fidelity sensing. After data acquisition is completed, the triggering signal is cleared, and the MOSFET turns off the Xnode. The communication of control messages between the ADXL362 and the Xnode is carried out via SPI bus through a four-wire connection. In addition to enable event-triggered sensing, the proposed switch should be designed to retain traditional functionality for periodic monitoring. Specifically, sensor nodes are operated on low duty cycles, and the base station can access the network of nodes at random to initiate operations or measurements in the network. To achieve this goal, a real time clock, DS3231, is employed in the proposed switch. When a user-defined period passes or at a specific time of day, the DS3231 sends a triggering signal which flips the MOSFET switch and turn on the Xnode. Then the node remains awake for a short period of time to listen for messages from the base station. Once a command is obtained, the node carries out the required task (e.g., sensing, battery check). The communication of control messages between the DS3231 and the Xnode is conducted through the I2C bus.

Figure 3a illustrates the design concept of the proposed switch. Five major components are implemented, including a trigger sensor ADXL362 (U1), a real time clock DS3231 (U2), an AND gate (U3), a latch (U4), and a MOSFET (U5). Interrupt pins from the ADXL362 and the DS3231 are connected to the MOSFET through the AND gate. This circuit enables the MOSFET to be triggered by either the 
real time clock or the trigger sensor. In addition, a latch component is added between the AND gate and the MOSFET, to keep the power supply stable. Figure 3b shows the realized PCB for the proposed switch. The five major components, as well as companion resistors and capacitors, are all soldered on the edge of top side. In some use cases such as downloading code to the board and debugging, the sensor platform should always be on and therefore the designed switch needs to be bypassed. To achieve this goal, a 2-pin jumper is added. When the two pins on the jumper are not connected, the proposed switch works as designed, otherwise, it is bypassed.

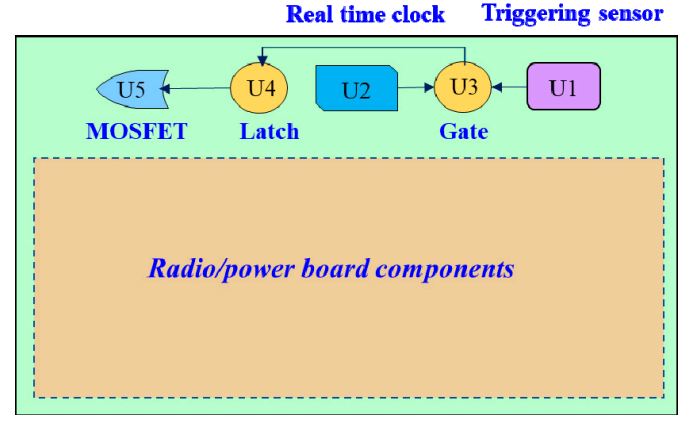

(a)

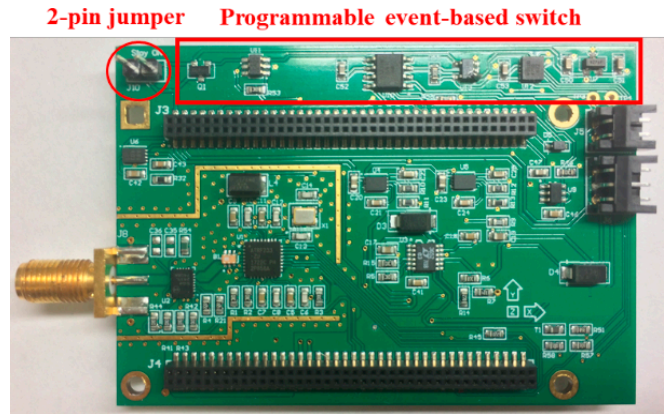

(b)

Figure 3. PCB design for the event-based switch: (a) design concept (b) realized PCB.

\subsubsection{Software Consideration}

In addition to hardware development of the prototype, an effective application framework is required to control the behavior of demand-based WSS to realize event-triggered sensing.

Figure 4 shows a flowchart of the application framework for the demand-based WSS. More specifically, when users turn on the physical switch of a main sensor platform, the Xnode first initializes itself and sends commands which contain configuration parameters (e.g., threshold, timers, and data buffer size) to the event-based switch discussed in the previous section. Once the commands have been received, the switch completes configuration of the device settings. Subsequently, the ADXL362 starts measurement in ultralow-noise mode, and the Xnode is turned off. If a sudden event occurs and the acceleration obtained in the ADXL362 exceeds the user-defined threshold, an interrupt pin, INT1 on the ADXL362 sends a trigger signal to turn on the Xnode. Concurrently, the ADXL362 saves 512 data samples into its FIFO buffer surrounding the onset of the event and waits for the Xnode to retrieve the data. The Xnode starts high-fidelity data acquisition using its built-in high-power high-accuracy MEMS accelerometer. When the event stops and the acceleration obtained in the ADXL362 is lower than a user-defined threshold for a certain period of time, the other interrupt pin, INT2, in the ADXL362 is triggered. Subsequently, the Xnode stops high-fidelity sensing. After sensing is completed, the Xnode reads data from the FIFO buffer of the ADXL362 and fuses it with the Xnode data. In addition, when the Xnode is busy with other tasks (e.g., data transmission of a previous event), but another sudden event occurs, the INT2 pin can be configured to interrupt undergoing tasks and force the Xnode to start high-fidelity sensing immediately. In addition, timing analysis results for each stage of a demand-based WSS are presented in the left of Figure 4.

For sudden events that are rare, e.g., earthquakes, the thresholds can be determined based on priori information about the sudden events that are monitored. The a priori information can be estimated by numerical analysis, the data in the history, or the measurement data in a preliminary test. For some events that occur frequently, e.g., railway bridge impacts from over-height vehicles, the thresholds can be determined adaptively, starting from a relatively low value during a "training phase" and then adjusted until the detection errors are minimized. A more comprehensive research regarding the triggering mechanism is the subject of future work to be addressed in the near future. 


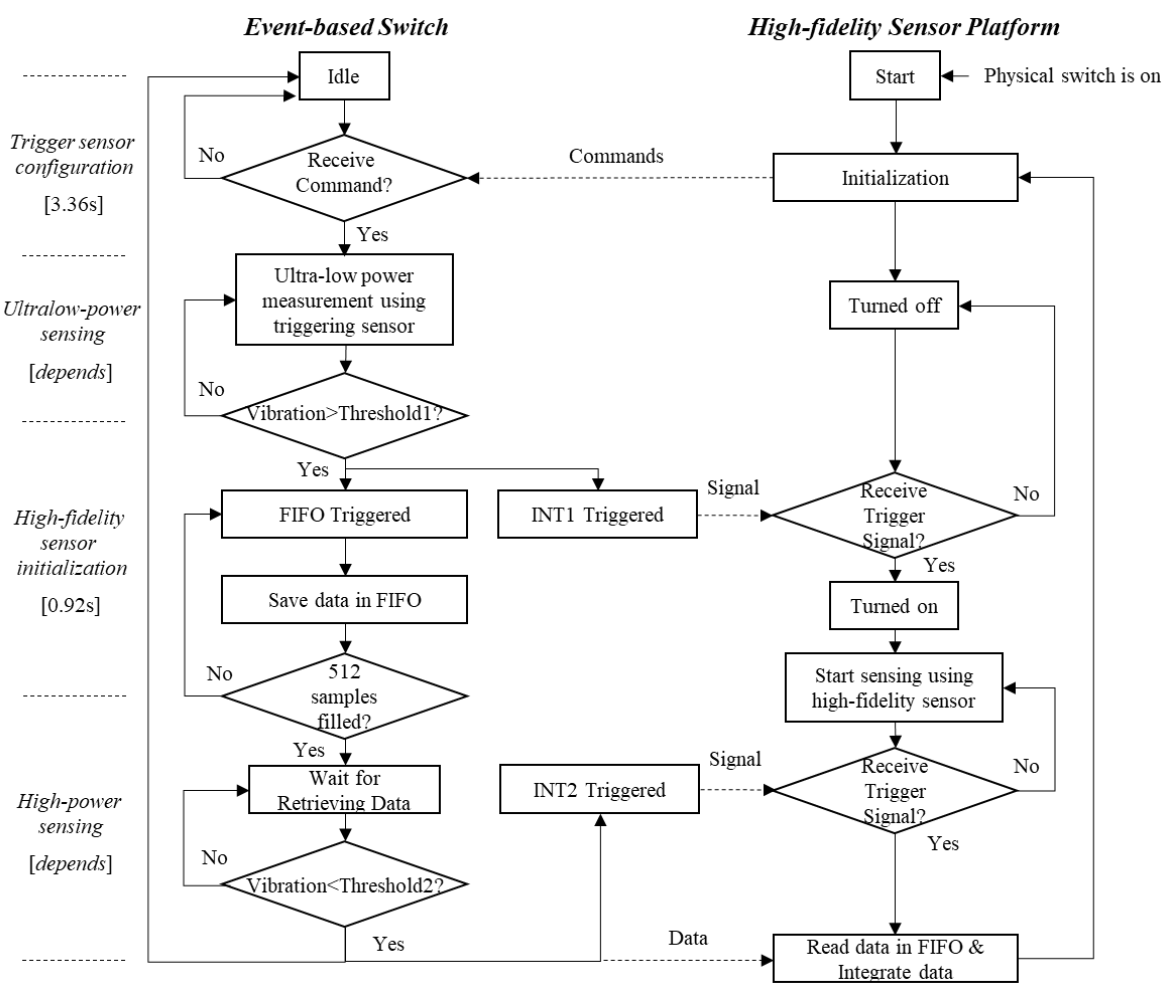

Figure 4. Flowchart of demand-based wireless smart sensors (WSS) for event-triggered sensing.

\subsubsection{Data Fusion of Trigger Sensor and Xnode Acceleration Record}

The objective of the demand-based WSS is to obtain the data from before the trigger event occurs until the structural accelerations stop. Specifically, the ADXL362 can record structural measurements surrounding the onset of a sudden event, whilst the Xnode starts sampling the data approximately $0.9 \mathrm{~s}$ after being triggered. Therefore, the ADXL362 data and the Xnode data must be synchronized and fused to produce a complete representation of the acceleration record. The following paragraphs describe the challenges encountered in this process, along with the associated resolutions.

To fuse the two overlapping data streams, two main challenges should be addressed, including (i) differences in the sampling rate between the ADXL362 and the Xnode, and (ii) synchronization error between the ADXL362 data and the Xnode data. More precisely, the first challenge results from the differences between the clock rates of the ADXL362 and the Xnode. The internal clock rate in the ADXL362 has a standard deviation of approximately 3\%. One approach might be to calibrate the ADXL362 incorporated in each Xnode; however, this approach is not practical, as the clock rate will change with temperature, invalidating the initial calibration. The second challenge is due to the variance in start-up time of the sensing task on the Xnode. As a result, a random offset will exist between the two data streams.

To tackle the challenges identified in the previous paragraph, the beginning of the Xnode data, which is overlapped with the ADXL362 data, was utilized to calibrate the entire ADXL362 data stream. Figure 5 shows a flowchart of this approach. More specifically, the ADXL362, acc adxl0, with a nominal sampling rate of $100 \mathrm{~Hz}$, were first up-sampled to $f_{s}(1000 \mathrm{~Hz})$. The last 400 data points of the acc $_{\text {adxl }}$. were chosen as $a c_{a d x l 1}$, which was assumed to be approximately overlapped with the beginning of the Xnode data. In the meantime, the Xnode data, $a c_{x n o d e 0}$, was sent through an 8-pole elliptic low-pass filter with a cutoff frequency of $50 \mathrm{~Hz}$, to have the same bandwidth with $a_{c c} c_{a d x l 0}$. The first 400 data points of $a c_{x n o d e 0}$ were considered as $a c_{\text {xnode } 1}$. Based on the datasheet of ADXL362 [26], the clock frequency deviation from the ideal value was within the range of $-10 \%$ and $10 \%$. Therefore, to find the 
actual sampling frequency of $a c c_{a d x l 1}$, exhaustive search was applied from $900 \mathrm{~Hz}$ to $1100 \mathrm{~Hz}$. For Step $i$, the estimated sampling frequency $\left(f_{e}\right)$ of the ADXL362 data was set as,

$$
f_{e}=1000-d f[i]
$$

where, $d f[i]=i-100, i \in[0,200]$. acc $a d x l 1$ was resampled from $f_{e}$ to $f_{s}$ using resampling-based approach [36]. Then, to estimate the synchronization error between $a_{c c} c_{a d x l 1}$ and $a_{c c_{x n o d e 1}}$, the cross-correlation between the two data segments was calculated. The optimal offset, $S E[i]$, was obtained, for which the cross-correlation reaches its maximum value. Afterwards, $a_{c c} a d x l 1$ was shifted by $S E[i]$, and then the data fusion error (Err) was calculated as,

$$
\operatorname{Err}[i]=\left\|a c c_{a d x l 1}-a c_{x n o d e 1}\right\|_{2}
$$

where, \|\|$_{2}$ represents Euclidean norm. After completing these steps, the best estimations of sampling frequency $f_{a}$ and synchronization error $S E_{a}$ were obtained in the step that achieves minimal Err. Subsequently, $f_{a}$ and $S E_{a}$ were applied to calibrate the original data set, $a c c_{a d x l 0}$. Finally, $a c c_{a d x l 0}$ and $a_{c c} x$ node0 were combined and down-sampled to $100 \mathrm{~Hz}$ for ensuing analysis.

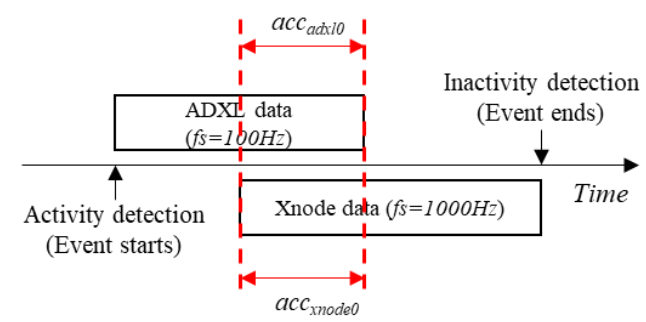

(a)
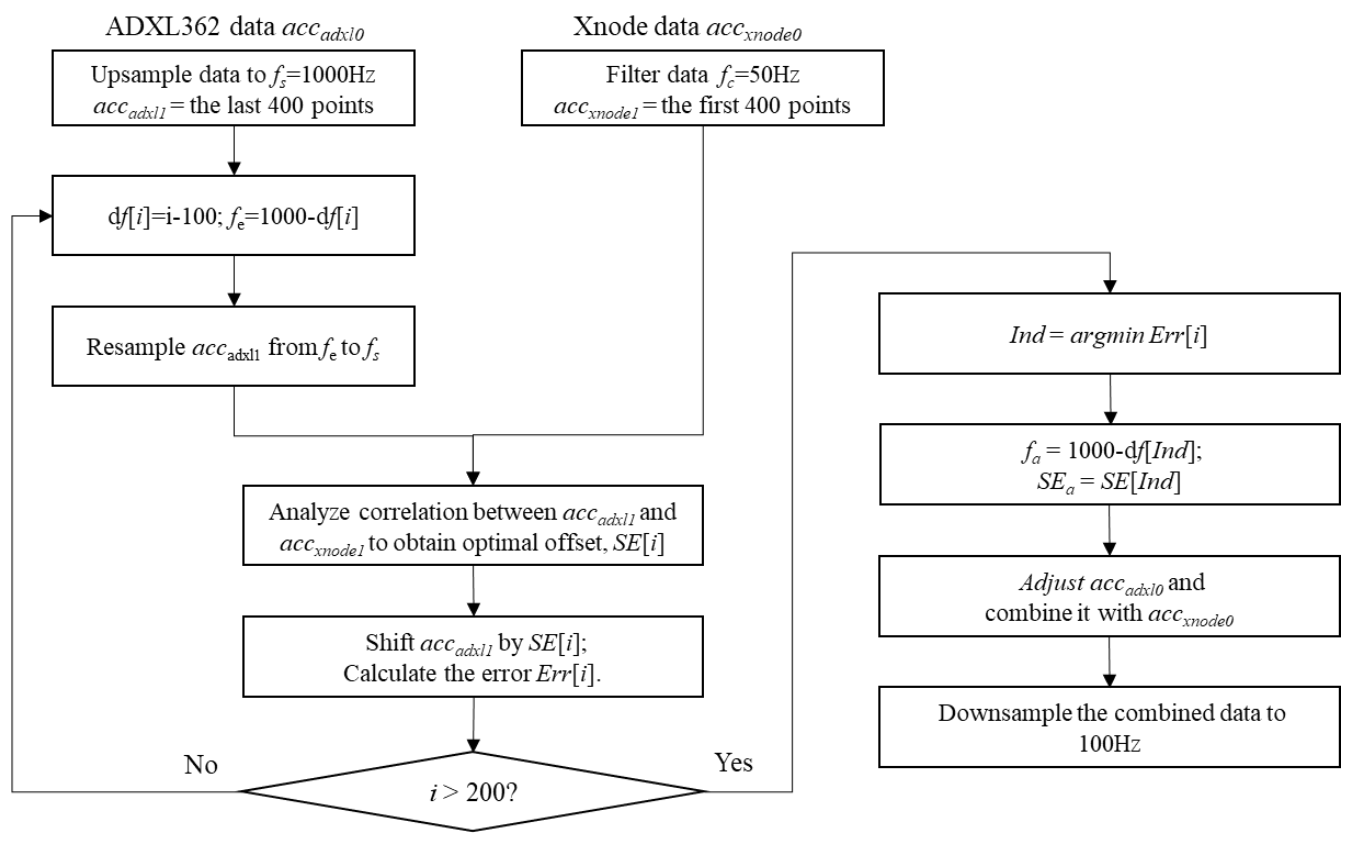

(b)

Figure 5. Post-sensing data fusion: (a) illustration of two data sources, (b) flowchart of data fusion strategy.

\section{Validation of the Demand-Based WSS Performance}

To validate the performance of the demand-based WSS, laboratory tests were carried out for data fusion and earthquake monitoring. The detailed test setup and results are presented in this section. 
The performance of the demand-based WSS is discussed, in terms of power consumption, sensing characteristics, and data quality for sudden event monitoring.

\subsection{Validation of Data Fusion}

A lab test was conducted to illustrate the challenge of data fusion between the ADXL362 data and the Xnode data. Specifically, a demand-based WSS was located at 10th floor of an 18-story building model shown in Figure 6. The ADXL362 was configured to capture samples at $100 \mathrm{~Hz}$, starting at $0.2 \mathrm{~s}$ before the triggered event and continuing until $1.5 \mathrm{~s}$ after the event. The event-triggering threshold was set to $150 \mathrm{mg}$, at which time, the Xnode was turned on and $1000 \mathrm{~Hz}$ high-fidelity measurement was started. To reduce false positives, two consecutive data points exceeding the threshold were required to cause triggering. In addition, a wired piezoelectric accelerometer, model PCB353B33, was installed on the same floor and sampled at a frequency of $128 \mathrm{~Hz}$. The acceleration from these sensors served as reference data. A sudden event was simulated by manually shaking the building model in horizontal direction.
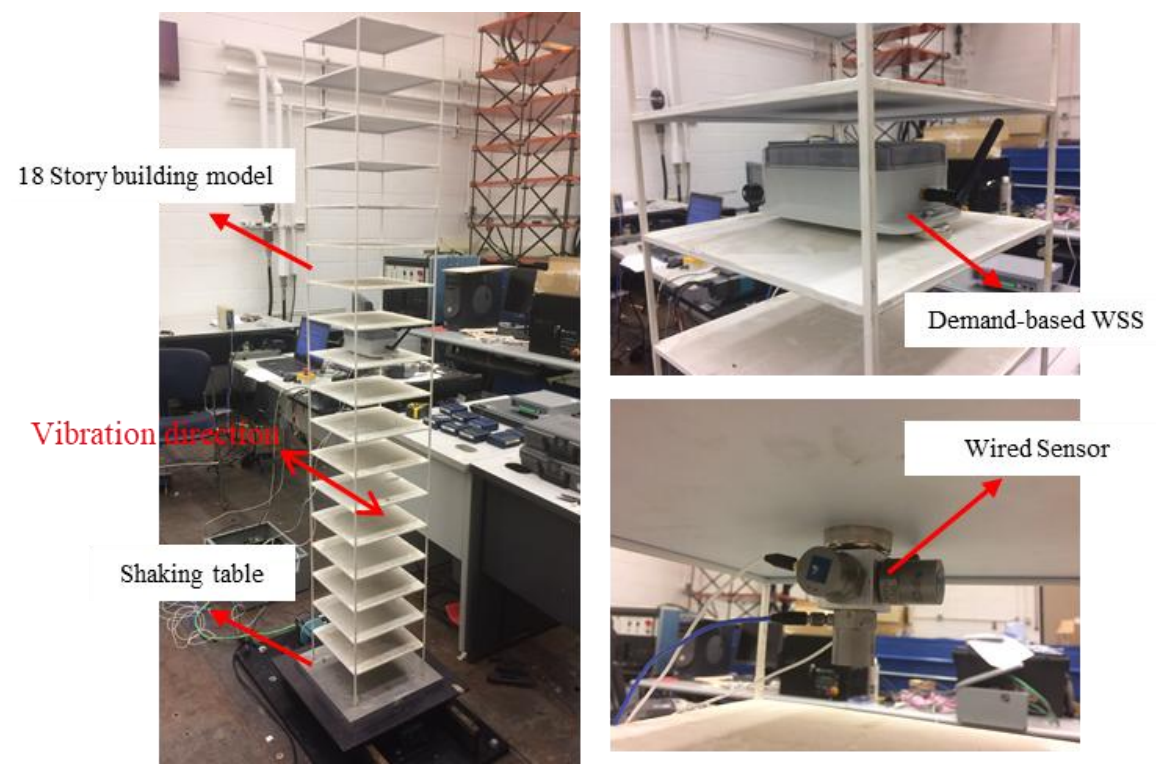

Figure 6. Experiment setup for a demand-based WSS.

To make a direct comparison in the time domain, the Xnode data and wired sensor data were sent through an 8-pole elliptic low-pass filter with a cutoff frequency of $50 \mathrm{~Hz}$, as displayed in Figure 7. The direction of acceleration measurement was the same with the vibration direction specified in Figure 6 . The vibration exceeded the threshold at $0.7 \mathrm{~s}$, and the event-based switch turned on the Xnode. The Xnode required $0.92 \mathrm{~s}$ for initialization. Fortunately, as shown in Figure $7 \mathrm{~b}$, acceleration data stored in the FIFO buffer of the ADXL362 was recorded during this period. Specifically, the ADXL362 data can be divided into three parts: (i) Part 1 is the pre-triggered data which is around $0.28 \mathrm{~s}$ in length; (ii) Part 2 is the data that cover the time where the Xnode is initializing; (iii) Part 3 is where the ADXL362 data overlaps with the Xnode data. The length of the data in Part 3 is approximately $0.6 \mathrm{~s}$. As shown in Figure 7b, the data obtained from the ADXL362 does not match well with the reference data from the wired sensors, because the sampling frequency of the ADXL362 was slightly smaller than $100 \mathrm{~Hz}$, which illustrates the first challenge mentioned in the Section 2.3.3. In addition, the time offset between the Xnode data (blue line in Figure 7a) and the ADXL362 data (red line in Figure 7b) must be accurately estimated to fuse these two data streams, which illustrates the second challenge of data fusion. 


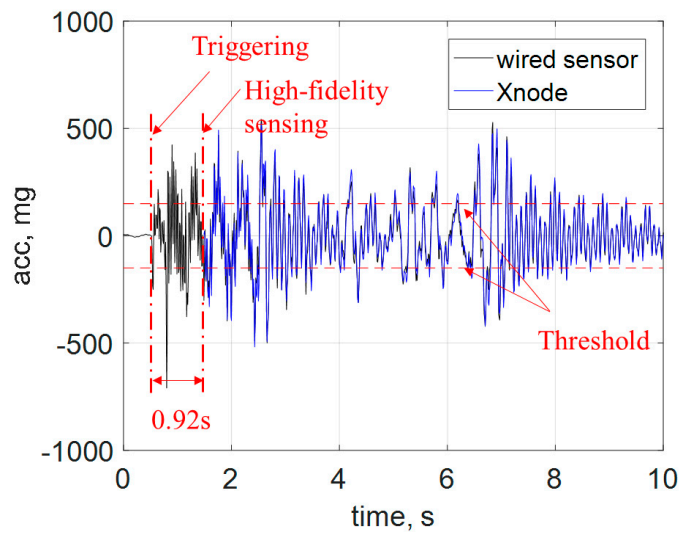

(a)

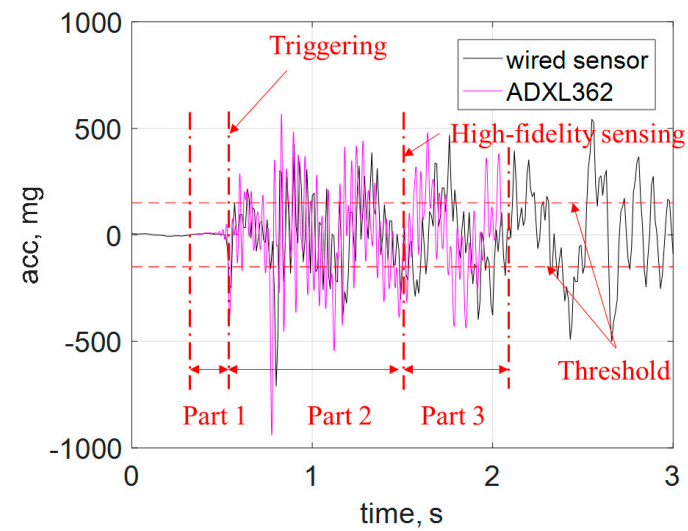

(b)

Figure 7. Time history data comparison: (a) Xnode measurement, (b) ADXL362 data buffer.

The data fusion strategy discussed in the previous section is applied to the test data. Figure 8 shows a comparison of time history data between sensor data from wired sensors and the fused data from the demand-based WSS. The excellent agreement demonstrates the ability of the proposed strategy to seamlessly capture the structural response subjected to a sudden event.

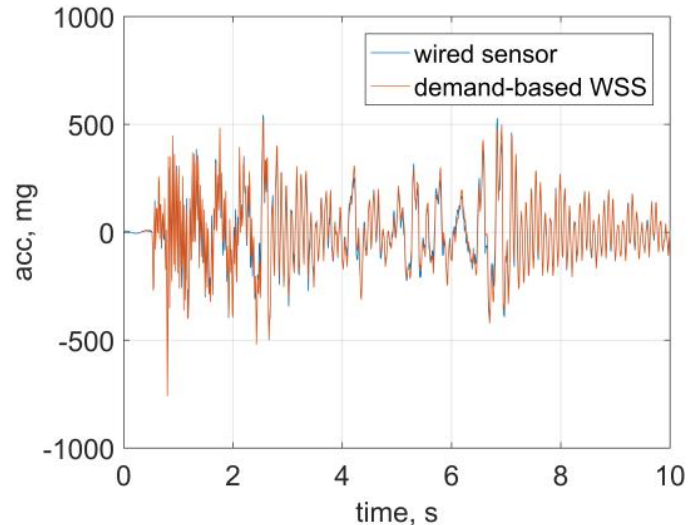

(a)

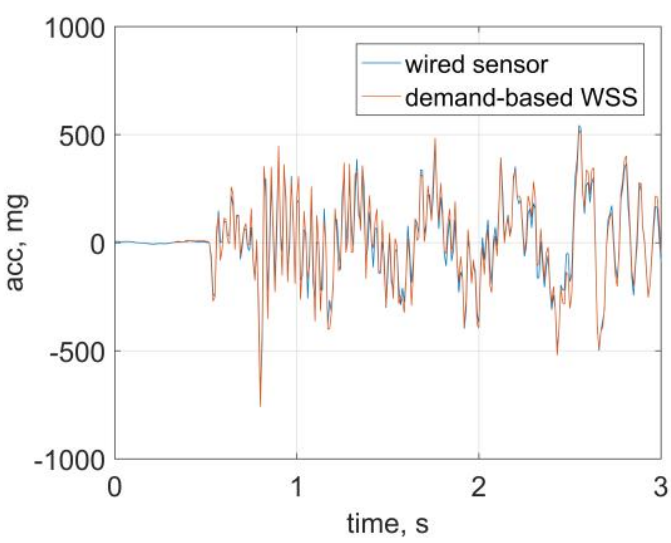

(b)

Figure 8. Results of data fusion: (a) time history data, (b) zoomed view (ADXL362 data).

\subsection{Earthquake Monitoring}

As a typical sudden event, an earthquake is transient and unpredictable, and the consequences can be catastrophic. Continuous efforts are required to develop cost-effective earthquake monitoring systems to mitigate the effect of earthquakes. Demand-based WSS have a significant potential to enable earthquake detection and rapid condition assessment of civil infrastructure, which was validated through a lab test in this section.

The test setup was the same with that in Section 3.1, as shown in Figure 6. The structure model was mounted on a uniaxial shaking table. This shaking table can simulate earthquakes in one horizontal direction, driving a $15 \mathrm{~kg}$ mass at $2.5 \mathrm{~g}$ with a maximum stroke of $\pm 7.5 \mathrm{~cm}$. The El Centro earthquake excitation was generated by the shaking table to represent a sudden event. The detection threshold for the event-based switch in demand-based WSS was configured as follows: the onset of event was detected when the acceleration was above $80 \mathrm{mg}$ over $0.02 \mathrm{~s}$, and the end of event was detected when the acceleration was below $40 \mathrm{mg}$ over $5 \mathrm{~s}$. Other configuration parameters are the same with the test in Section 3.1, such as sampling frequencies and filter parameters.

A segment of $90 \mathrm{~s}$ recorded time history is shown in Figure 9a. As can be seen in the zoomed view of the time history data (Figure $9 \mathrm{~b}, \mathrm{c}$ ), the ground motion started at $10.7 \mathrm{~s}$, but the vibration in the 
beginning was very small. From $11.79 \mathrm{~s}$ to $11.80 \mathrm{~s}$, two consecutive acceleration samples obtained by the trigger accelerometer exceeded $80 \mathrm{mg}$. As a result, the event-based switch turned on the demand-based WSS immediately and the WSS started high-fidelity measurement. The acceleration became smaller than $40 \mathrm{mg}$ after $54.50 \mathrm{~s}$. Approximately $5 \mathrm{~s}$ later, the event-based switch stopped the high-fidelity sensing. Furthermore, Figure $9 \mathrm{~d}$ shows the power spectral density (PSD) in the frequency domain. The excellent agreement between the results of wired sensors and the demand-based WSS in the both time and frequency domain demonstrates the ability of the proposed WSS to detect the earthquake and capture the accurate structural response during earthquakes in a timely and efficient manner.

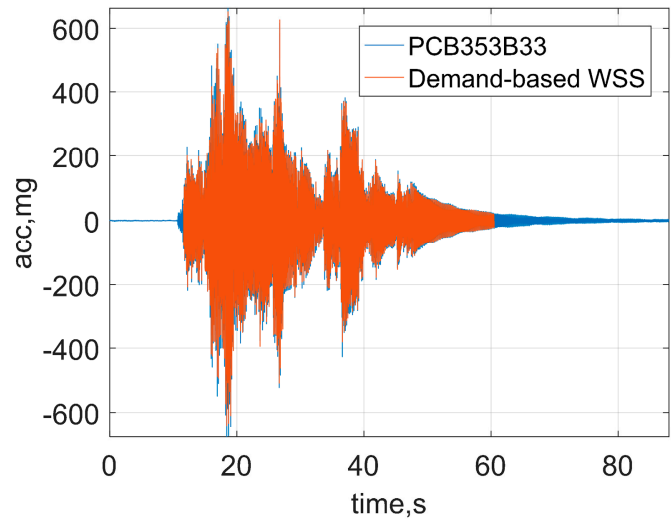

(a)

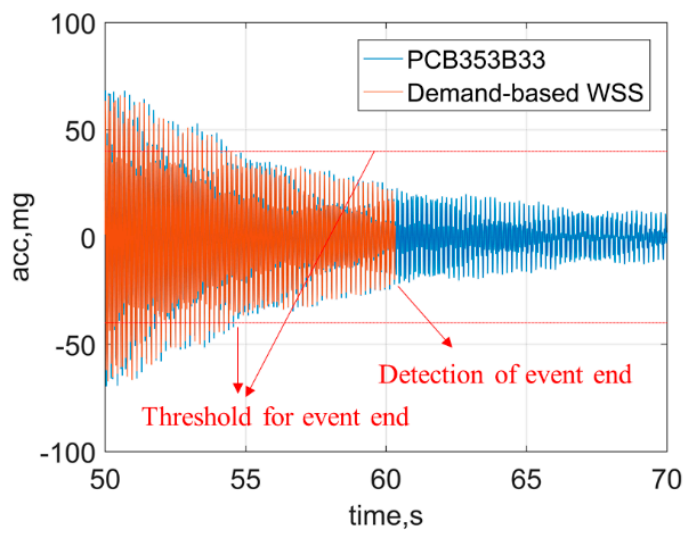

(c)

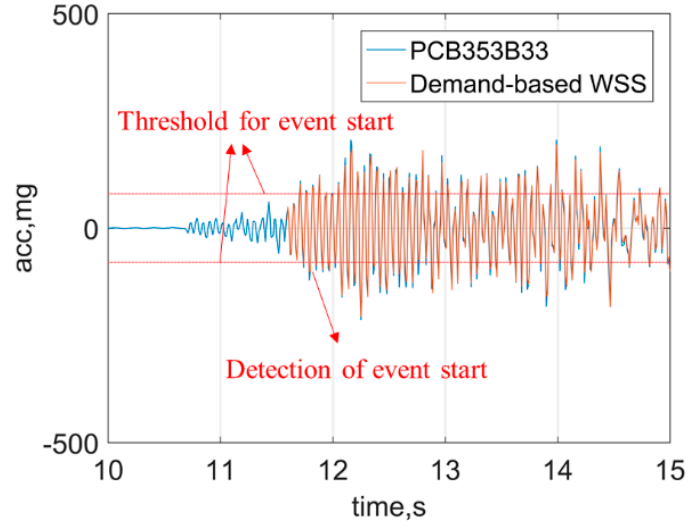

(b)

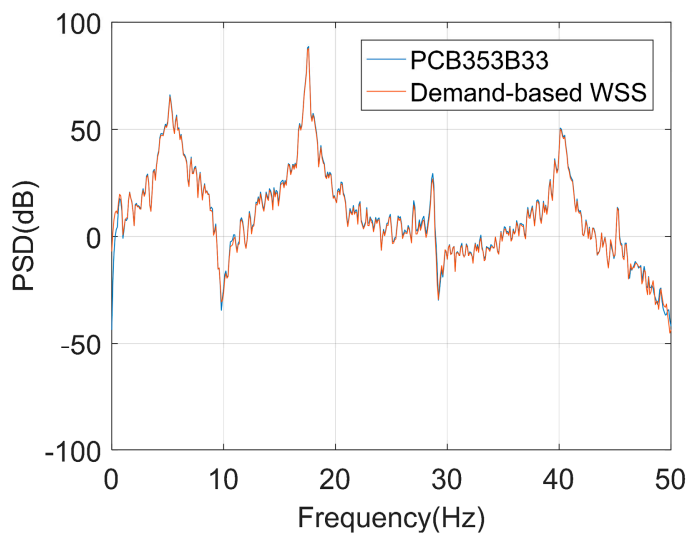

(d)

Figure 9. Test results of earthquake monitoring: (a) time history data, (b) zoomed view of time history data for event start, (c) zoomed view of time history data for event end, (d) power spectral density (PSD) data.

\subsection{Evaluation and Discussion}

In the lab tests described in previous sections, the attractive performance of the demand-based WSS was successfully validated to detect sudden events and provide high-quality sensing data for SHM analysis.

(1) Power consumption tests showed that the proposed WSS has a current draw of only $365 \mu \mathrm{A}$ when no sudden event occurred, but the power consumption of the original Xnode sensor platform is approximately $170 \mathrm{~mA}$ during sensing. Considering that sudden events are rare and short-duration, most of the time the demand-based WSS deployed on a structure is in low-power measurement mode. Therefore, if using a $3.7 \mathrm{~V} \mathrm{DC}, 10,000 \mathrm{mAh}$, rechargeable lithium polymer battery, employing the proposed WSS can extend the lifetime of always-on monitoring from three days to over three years using a single lithium battery. This feature helps to successfully 
detect the occurrence of sudden events with minimal power budget in long-term monitoring. In addition, the current draw in each operation associated with duration for a demand-based WSS was determined experimentally and shown in Figure 10, in which the majority component of power consumption is sensing.

(2) The data obtained from the demand-based WSS is high-quality, matching well with the data from wired piezoelectric accelerometers. In particular, high-fidelity sensing enables 24-bit sensing resolution and over $1 \mathrm{kHz}$ sampling rates. This feature helps to conduct structural condition assessments accurately under sudden events.

(3) The test results show that, when an event occurs, a seamless transition from the low-power sensing to high-fidelity measurement is carried out, without losing any data about the event.

These three features demonstrate that the proposed WSS satisfies the demands of sudden event monitoring.

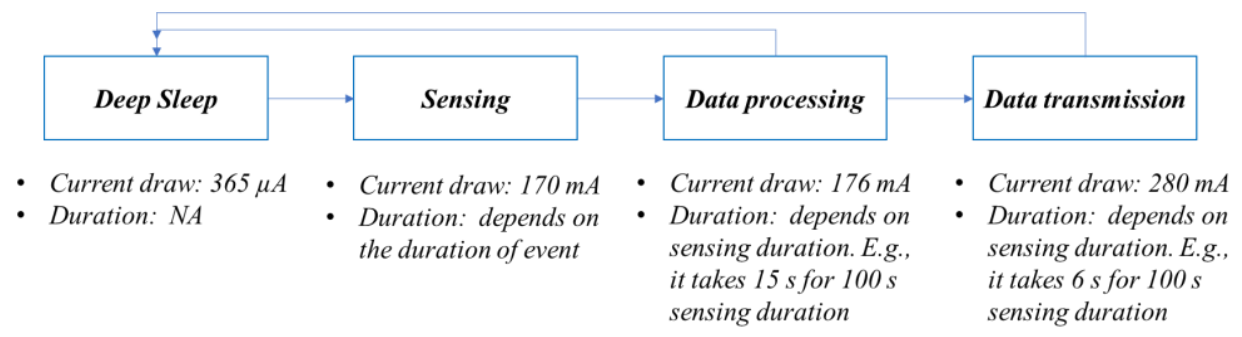

Figure 10. Flowchart of event-triggered sensing regarding current draw and duration for each operation.

\section{Field Application}

To further validate system performance, a field test was conducted on a steel railroad bridge north of Champaign, Illinois. Having vibration data while in-service trains traverse the bridge is useful to assess the bridge condition [37]. Train events have similar features to sudden natural events, e.g., unpredictability due to uncertain train schedules, but occur more frequently and therefore provide a convenient test platform. A demand-based WSS was deployed on the bottom side of a bridge girder. Simultaneously, wired sensors, model PCB353B33, were selected as reference sensors and deployed close to the WSS (see Figure 11). A detection threshold was configured to be the same as the test in Section 3.2. To avoid signal saturation, the measurement range of the trigger accelerometer was set to the maximum value of 8 g. At 10:52:06 a.m. on 7 May 2019, an Amtrak passenger train passed by the bridge.

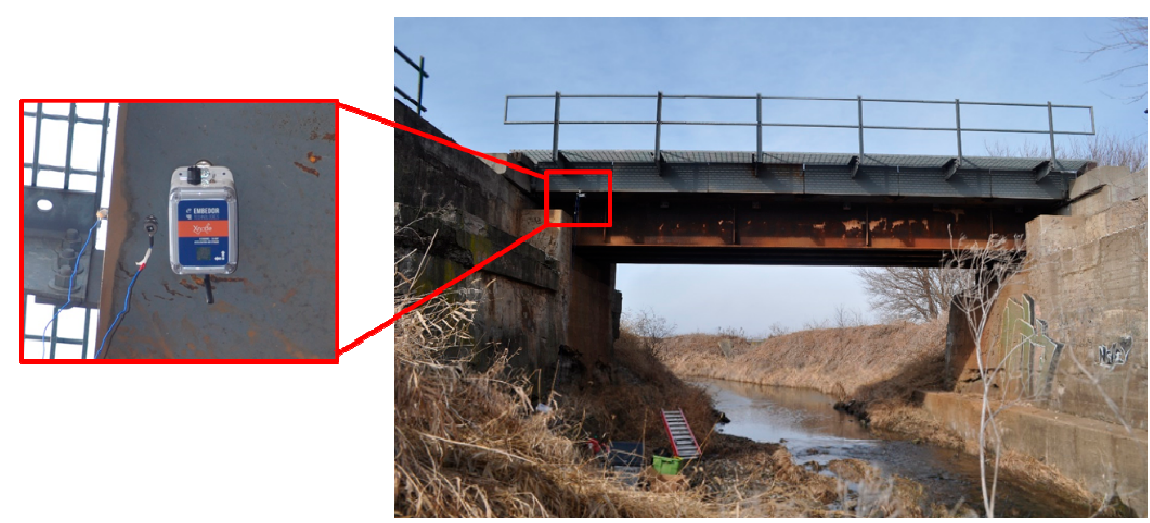

Figure 11. Field application of the demand-based WSS.

Figure 12a-d shows the raw acceleration data of the bridge in vertical direction. The train came to the bridge at $121 \mathrm{~s}$ and left at $128 \mathrm{~s}$. The event had a short duration of $7 \mathrm{~s}$, and it was successfully 
detected by the demand-based WSS. Figure 12e shows the PSD data. Some slight discrepancies between the data from two sensors are possibly due to the different locations of the sensors. In sum, good agreement can be observed between the wired sensor and the demand-based WSS both in the time and frequency domain, demonstrating that the new WSS can capture the sudden event and obtain high-fidelity measurement in real applications.

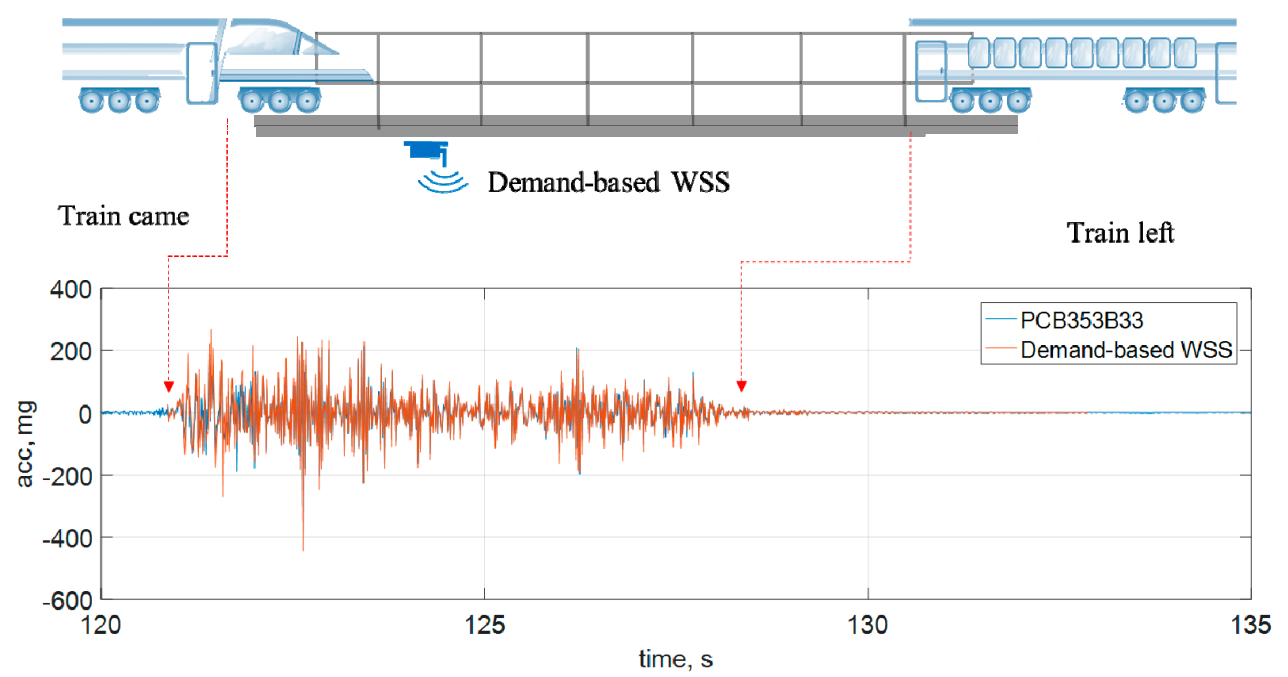

(a)

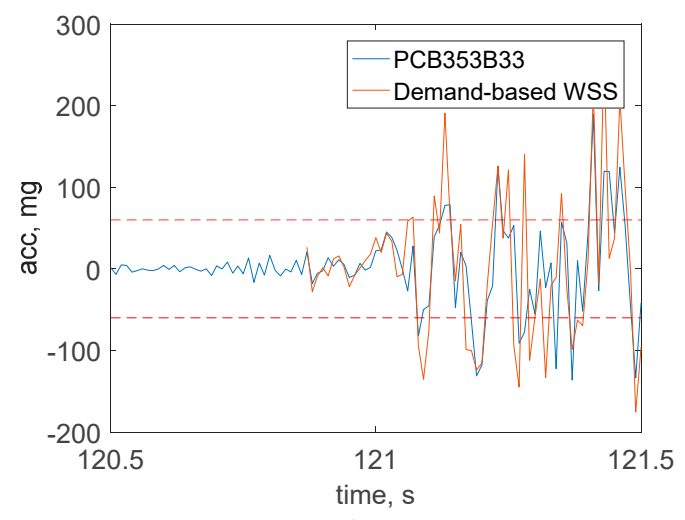

(b)

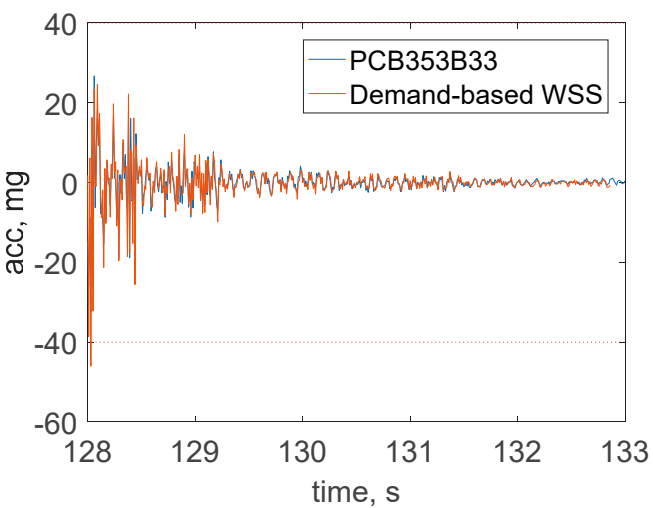

(d)

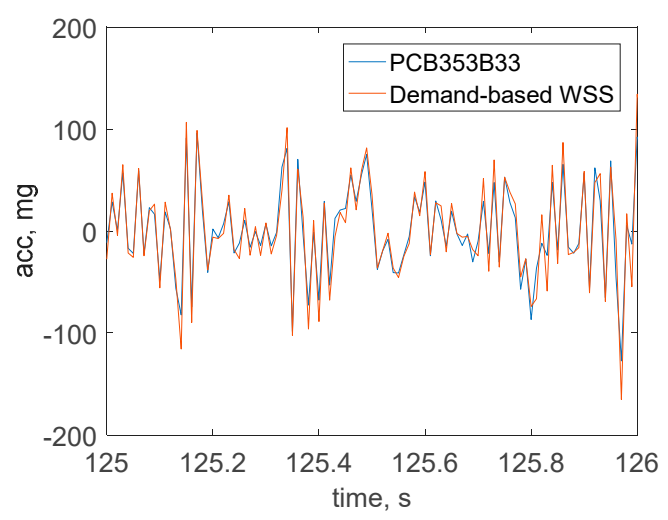

(c)

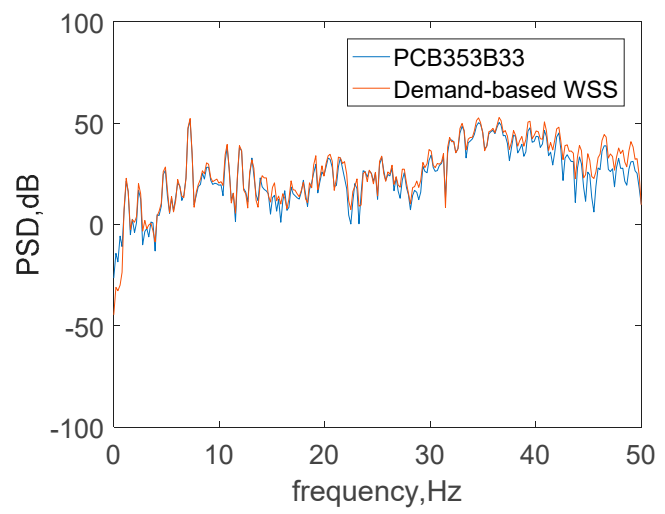

(e)

Figure 12. Test results on a railroad bridge: (a) time history data, (b) zoomed view of event starts (ADXL362 data), (c) zoomed view of event data (Xnode data), (d) zoomed view of event ends (Xnode data), (e) PSD data. 


\section{Conclusions}

This paper presented the design of demand-based WSS to meet the application requirements of sudden event monitoring. The proposed WSS mainly consist of a unique programmable event-based switch and a powerful high-fidelity WSS platform. In particular, the event-based switch is built on a trigger accelerometer which allows the new WSS to measure the structural response in ultralow-power in long term, so as not to miss sudden events. In addition, the software of event-triggered sensing and data fusion is implemented. The performance of the proposed WSS is evaluated through the lab tests of earthquake monitoring and a field application on a railroad bridge. The test results show that the proposed WSS can continuously monitor structural response with minimal power budget, and hence detect the occurrence of the sudden event with the smallest delay. Besides detecting sudden events, the proposed WSS have the excellent features of high sampling rates and sensing resolution, which finally helps to provide high-quality data in sudden events for rapid condition assessment of civil infrastructure. Moreover, the proposed WSS are powerful and versatile not only for sudden events (e.g., earthquakes), but also for autonomous monitoring of other general events (e.g., bridge/highway overloads).

For large-scale structures, one demand-based WSS is not sufficient, and a network of nodes are needed for a meaningful characterization of the structural response. When subjected to a sudden event, each node may be triggered independently to initiate measurement at slightly different times due to varying response levels in the structure. Future work will address the challenges encountered for a network of demand-based WSS under sudden events. For example, one critical issue is to synchronize data from different sensor nodes without introducing delay of event-triggered sensing.

Author Contributions: Conceptualization, Y.F.; methodology, Y.F. and T.H.; software, Y.F. and T.H.; validation, Y.F., T.H., K.M. and B.F.S.J.; formal analysis, Y.F.; investigation, Y.F. and T.H.; resources, K.M. and B.F.S.J.; data curation, Y.F.; writing-original draft preparation, Y.F.; writing-review and editing, T.H., K.M., J.R.K., D.Z., B.F.S.J.; visualization, Y.F.; supervision, K.M. and B.F.S.J.; project administration, B.F.S.J. and J.R.K.; funding acquisition, J.R.K. and D.Z.

Funding: This research was funded in part by the Nazarbayev University Research Fund under Grant \#SOE2017003, ZJU-UIUC Institute Research under Grant \#ZJU083650, Federal Railroad Administration under Grant \#DTFR53-17-C00007, as well as China Scholarship Council scholarship.

Acknowledgments: The authors gratefully acknowledge the support of this research by the Federal Railroad Administration, Canadian National Railway, and Sandro Scola, as well as the China Scholarship Council.

Conflicts of Interest: The authors declare no conflict of interest.

\section{References}

1. Garner, A.C.; Huff, W.A. The wreck of Amtrak's Sunset Limited: News coverage of a mass transport disaster. Disasters 1997, 21, 4-19. [CrossRef] [PubMed]

2. Yamazaki, F.; Katayama, T.; Yoshikawa, Y. On-line damage assessment of city gas networks based on dense earthquake monitoring. In Proceedings of the 5th U.S. National Conference on Earthquake Engineering, Chicago, IL, USA, 10-14 July 1994; Volume 4, pp. 829-837.

3. Yamazaki, F.; Motomura, H.; Hamada, T. Damage assessment of expressway networks in Japan based on seismic monitoring. In Proceedings of the 12th World Conference on Earthquake Engineering, Auckland, New Zealand, 30 January-4 February 2000.

4. Celebi, M. Real-time seismic monitoring of the New Cape Girardeau Bridge and preliminary analyses of recorded data: An overview. Earthq. Spectra 2006, 22, 609-630. [CrossRef]

5. Okada, K.; Nakamura, Y.; Saruta, M. Application of earthquake early warning system to seismic-isolated buildings. J. Disaster Res. 2009, 4, 242-250. [CrossRef]

6. Rice, J.A.; Mechitov, K.; Sim, S.H.; Nagayama, T.; Jang, S.; Kim, R.; Spencer, B.F.; Agha, G.; Fujino, Y. Flexible smart sensor framework for autonomous structural health monitoring. Smart Struct. Syst. 2010, 6, 423-438. [CrossRef]

7. Caicedo, J.M.; Clayton, E.; Dyke, S.J.; Abe, M.; Tokyo, J. Structural health monitoring for large structures using ambient vibrations. In Proceedings of the ICANCEER Conference, Hong Kong, China, 15-20 August 2002. 
8. Cheng, L.; Pakzad, S.N. Agility of wireless sensor networks for earthquake monitoring of bridges. In Proceedings of the 2009 IEEE Sixth International Conference on Networked Sensing Systems (INSS), Pittsburgh, PA, USA, 17-19 June 2009.

9. Guo, S.; Kim, S.M.; Zhu, T.; Gu, Y.; He, T. Correlated flooding in low-duty-cycle wireless sensor networks. In Proceedings of the 2011 19th IEEE International Conference on Network Protocols, Vancouver, BC, Canada, 17-20 October 2011; pp. 383-392.

10. Potenza, F.; Federici, F.; Lepidi, M.; Gattulli, V.; Graziosi, F.; Colarieti, A. Long-term structural monitoring of the damaged Basilica S. Maria di Collemaggio through a low-cost wireless sensor network. J. Civ. Struct. Health Monit. 2015, 5, 655-676. [CrossRef]

11. Miller, T.I.; Spencer, B.F., Jr.; Li, J.; Jo, H. Solar Energy Harvesting and Software Enhancements for Autonomous Wireless Smart Sensor Networks; NSEL Report No. 022; University of Illinois at Urbana-Champaign: Urbana, IL, USA, 2010.

12. Jang, S.; Jo, H.; Cho, S.; Mechitov, K.A.; Rice, J.A.; Sim, S.H.; Jung, H.-J.; Yun, C.-B.; Spencer, B.F., Jr.; Agha, G. Structural health monitoring of a cable-stayed bridge using smart sensor technology: Deployment and evaluation. Smart Struct. Syst. 2010, 6, 439-459. [CrossRef]

13. Park, J.W.; Jung, H.J.; Jo, H.; Spencer, B.F., Jr. Feasibility study of micro wind turbines for powering wireless sensors in a cable-stayed bridge. Energies 2012, 5, 3450-3464. [CrossRef]

14. Lu, X.; Wang, P.; Niyato, D.; Han, Z. Resource allocation in wireless networks with RF energy harvesting and transfer. IEEE Netw. 2015, 29, 68-75. [CrossRef]

15. Lu, X.; Wang, P.; Niyato, D.; Kim, D.I.; Han, Z. Wireless networks with RF energy harvesting: A contemporary survey. IEEE Commun. Surv. Tutor. 2015, 17, 757-789. [CrossRef]

16. Jalsan, K.E.; Soman, R.N.; Flouri, K.; Kyriakides, M.A.; Feltrin, G.; Onoufriou, T. Layout optimization of wireless sensor networks for structural health monitoring. Smart Struct. Syst. 2014, 14, 39-54. [CrossRef]

17. Rault, T.; Bouabdallah, A.; Challal, Y. Energy efficiency in wireless sensor networks: A top-down survey. Comput. Netw. 2014, 67, 104-122. [CrossRef]

18. Lu, G.; De, D.; Xu, M.; Song, W.Z.; Cao, J. TelosW: Enabling ultra-low power wake-on sensor network. In Proceedings of the 2010 IEEE Seventh International Conference on Networked Sensing Systems (INSS), Kassel, Germany, 15-18 June 2010.

19. Polastre, J.; Szewczyk, R.; Culler, D. Telos: Enabling ultra-low power wireless research. In Proceedings of the 4th International Symposium on Information Processing in Sensor Networks, Boise, ID, USA, 15 April 2005; p. 48.

20. Sutton, F.; Da Forno, R.; Gschwend, D.; Lim, R.; Gsell, T.; Beutel, J.; Thiele, L. A Heterogeneous System Architecture for Event-triggered Wireless Sensing. In Proceedings of the 2016 15th ACM/IEEE International Conference on Information Processing in Sensor Networks (IPSN), Vienna, Austria, 11-14 April 2016.

21. Bischoff, R.; Meyer, J.; Enochsson, O.; Feltrin, G.; Elfgren, L. Event-based strain monitoring on a railway bridge with a wireless sensor network. In Proceedings of the 4th International Conference on Structural Health Monitoring of Intelligent Infrastructure, Zurich, Switzerland, 22-24 July 2009; Volume 2224, p. 7482.

22. Liu, Y.; Voigt, T.; Wirström, N.; Höglund, J. ECOVIBE: On-Demand Sensing for Railway Bridge Structural Health Monitoring. IEEE Internet Things J. 2018. [CrossRef]

23. Popovic, N.; Feltrin, G.; Jalsan, K.E.; Wojtera, M. Event-driven strain cycle monitoring of railway bridges using a wireless sensor network with sentinel nodes. Struct. Control Health Monit. 2017, 24, e1934. [CrossRef]

24. Hung, S.L.; Ding, J.T.; Lu, Y.C. Developing an energy-efficient and low-delay wake-up wireless sensor network-based structural health monitoring system using on-site earthquake early warning system and wake-on radio. J. Civ. Struct. Health Monit. 2018, 1-13. [CrossRef]

25. Dorvasha, S.; Lib, X.; Pakzada, S.; Chengb, L. Network architecture design of an agile sensing system with sandwich wireless sensor nodes. Proc. SPIE 2012, 8345, 83450H.

26. Analog Devices. ADXL362 Datasheet. 2016. Available online: http://www.analog.com/media/en/ technical-documentation/data-sheets/ADXL362.pdf (accessed on 18 November 2018).

27. Analog Devices. ADXL372 Datasheet. 2017. Available online: http://www.analog.com/media/en/ technical-documentation/data-sheets/adxl372.pdf (accessed on 18 November 2018).

28. STMicroelectronics. LIS3DSH Datasheet. 2017. Available online: https://www.st.com/resource/en/ datasheet/lis3dsh.pdf (accessed on 18 November 2018). 
29. InvenSense. MPU-6050 Datasheet. 2013. Available online: https://www.invensense.com/products/motiontracking/6-axis/mpu-6050/ (accessed on 18 November 2018).

30. Kane, M.; Zhu, D.; Hirose, M.; Dong, X.; Winter, B.; Häckell, M.; Lynch, J.P.; Wang, Y.; Swartz, A. Development of an extensible dual-core wireless sensing node for cyber-physical systems. In Proceedings of the Sensors and Smart Structures Technologies for Civil, Mechanical, and Aerospace Systems 2014, San Diego, CA, USA, 9-13 March 2014; Volume 9061, p. 90611U.

31. BeanAir. AX-3D Datasheet. 2016. Available online: http://www.beanair.com/wa_files/Datasheet-WirelessAccelerometer-BeanDevice-AX-3D.pdf (accessed on 18 November 2018).

32. Libelium. Waspmote v15 Datasheet. 2016. Available online: http://www.libelium.com/downloads/ documentation/waspmote_datasheet.pdf (accessed on 18 November 2018).

33. LORD Sensing. G-Link-200 Datasheet. 2017. Available online: http://www.microstrain.com/sites/default/ files/g-link-200_datasheet_8400-0102_2_0.pdf (accessed on 18 November 2018).

34. Spencer, B.F., Jr.; Park, J.W.; Mechitov, K.A.; Jo, H.; Agha, G. Next Generation Wireless Smart Sensors toward Sustainable Civil Infrastructure. In Proceedings of the Sustainable Civil Engineering Structures and Construction Materials (SCESCM 2016), Bali, Indonesia, 5-7 September 2016.

35. Fu, Y.G.; Mechitov, K.A.; Hoskere, V.; Spencer, B.F., Jr. Development of RTOS-based wireless SHM system: Benefits in applications. In Proceedings of the International Conference on Smart Infrastructure and Construction, Cambridge, UK, 27-29 June 2016.

36. Nagayama, T.; Spencer, B.F., Jr. Structural Health Monitoring Using Smart Sensors; Newmark Structural Engineering Laboratory, University of Illinois at Urbana-Champaign: Urbana, IL, USA, 2007.

37. Moreu, F.; Kim, R.E.; Spencer, B.F., Jr. Railroad bridge monitoring using wireless smart sensors. Struct. Control Health Monit. 2017, 24, e1863. [CrossRef]

(C) 2018 by the authors. Licensee MDPI, Basel, Switzerland. This article is an open access article distributed under the terms and conditions of the Creative Commons Attribution (CC BY) license (http:/ / creativecommons.org/licenses/by/4.0/). 